\section{Benefits from SAX}

SIR - We feel obliged to respond to your article (Nature 366, 101; 1993) on the $\mathrm{X}$-ray satellite SAX, which contains a number of mere statements about the competitiveness of SAX - or rather the lack of it - without context or backing.

One of the most prominent features of the X-ray sky is its extreme variability, with many transient and burst-like phenomena. To study the astrophysics of compact stellar X-ray binaries and nuclei of active galaxies for example, a consistent astrophysical picture can be obtained only by observations over the widest possible $\mathrm{X}$-ray bandwidth, and with a certain spectral resolving power.

The unique feature of the SAX satellite is that it carries a scientific payload comprising a balanced set of instruments that are mutually well tuned in sensitivity and spectral resolution so as to provide simultaneity over an unprecedented combination of X-ray bandwidth (three orders of magnitude $0.2-200 \mathrm{keV}$ ) and spectral resolving power $(\lambda / \Delta \lambda$ typically $10-20)$. This is achieved by four bore-sighted X-ray (imaging) spectrometers with a typical field of view of $1^{\circ}$ and with substantial overlap, which allows for adequate normalization of sensitivity and the cancellation of undesirable systematic effects in individual instruments. Moreover, careful selection of the SAX low-Earth (near equatorial) orbit permits operation in an environment of stable and minimal radiation-induced background noise. Many of the instruments are novel.

Second, SAX's wide-band spectral coverage will also be linked to its ability to detect and monitor highly variable and transient $X$-ray sources with the aid of two wide-field $\left(35^{\circ}\right) \mathrm{X}$-ray cameras. This provides a unique potential for dynamical studies of transient and non-periodic phenomena in the X-ray sky. The wide-field $\mathrm{X}$-ray cameras on SAX, a prototype of which is still operational on the Russian space station Mir, are the most sophisticated shadow-mask cameras so far developed in terms of field coverage over angular resolving power $\left(3 \times 10^{5}\right.$ pixels $)$ and sensitivity.

If compared to the impressive performance of the two major X-ray satellites now in operation, ROSAT and ASCA, SAX is both competitive and complementary. ROSAT now mainly focuses on deep imaging and mapping of sources with low X-ray surface brightness limited to soft $\mathrm{X}$-rays $(<2.5 \mathrm{keV})$. The ASCA mission, launched this year, is directed towards medium-resolution spectroscopic studies of X-ray sources in the $0.5-10 \mathrm{keV}$ passband, albeit with higher throughput and somewhat better energy resolution, but with lower spatial resolution than the SAX instrument covering this part of the spectrum. SAX, on the other hand, aims primarily at attaining high-quality spectral variation data on relatively bright variable and transient sources including the high energy continuum ( $>10 \mathrm{keV})$, which is beyond the reach of ROSAT and ASCA.

In summary, SAX is a sophisticated satellite with state-of-the-art instrumentation, which, with its unique combination of medium spatial and spectral resolution and broad band energy response, will provide unique insights into a wide range of astrophysical objects.

We wish to emphasize, however, that the discussion in your article of the evolution in costs of SAX is confusing without a proper reference. For external reasons, the project has undergone substantial evolution and delay since its inception in 1992, such as the transfer from a shuttle flight free of charge to a fully charged Atlas Centaur, the associated spacecraft redesign and the inclusion of a new ground segment infrastructure; moreover, the figures quoted in the article refer to economic conditions covering a period of more than ten years; without a proper backdating, comparison is meaningless.

I write on behalf of the SAX Science Steering Committee, of which I am chairman and whose members are J. A. M. Bleeker (Space Research Organization, Utrecht, The Netherlands); G. Boella (CNR Istituto Fisica Cosmica e Tecnologie Relative, Milan, Italy); G. Di Cucco (Istituto Tecnologie e Studio Radioazioni Extraterrestri, Bologna, Italy); G. C. Perola (Istituto Osservatorio Astronomico Universita 'La Sapienza', Rome, Italy); A. Preite-Martinez (CNR Istituto Astrofisica Spaziale, Frascati, Italy); M. Salvati (Osservatorio Astrofisico Arcetri, Firenze, Italy); B. G. Taylor (ESTEC/ ESA, Noordwijk, The Netherlands); E. P. J. Van den Heuvel (Astronomical Institute 'A. Pannekoek', University of Amsterdam, The Netherlands).

L. Scarsi

Istituto Fisica Cosmica

e Applicazioni Informatica/CNR,

ViaM. Stabile 172

90138 Palermo, Italy

SIR - I basically agree with Professor Scarsi in the preceding letter about the scientific potential of SAX. This X-ray astronomy satellite will combine a number of instruments which, when taken separately, do not surpass the capabilities of current instruments on MIR-KVANT, Granat, ROSAT, GRO, ASCA and XTE. But taken together, the SAX instruments will be very useful because they combine broad energy band on the one hand with the richness and variability of the X-ray sky on the other.

The main problems of SAX are political and managerial. The delays caused by a weak management have had a direct impact on the cost as well as on the scientific potential. The latter is being eroded simply because the frontline of X-ray astronomy is progressing. It is imperative to strengthen the project management and control in order to save SAX.

\section{J. Trumper}

Max-Planck-Institut

für Extraterrestrische Physik, 85740 Garching, Germany

\section{'Wrong' ideas}

SIR - Jonathan Cowie (Nature 365, 202; 1993), from the fact that only 5.7 per cent of authors have difficulties with publication of their work in peer-reviewed journals, drew the conclusion that most landmark works (perhaps 94.3 per cent) were and are "readily considered by the scientific community". But the number of landmark works originally rejected is too large to be explained by chance.

The problem of unorthodox ideas and work in science is becoming more acute with the growth of the institutionalization of science, with the concomitant rise of conformism and uniformity of thinking. The mechanism is simple: if a scientist is developing an idea in contradiction with accepted dogma, the chance of obtaining funds or of publishing a paper is small.

The root of the problem seems to lie in the general unconscious acceptance of the philosophy of naive realism, the basis of which is the belief that 'absolute truth' exists and is reflected, at least partially, by 'true' theories; the role of science is to distinguish the true theory from several rival ones. After the 'true' theory is selected and considered to be proved, all efforts and funds are concentrated on its development; any attempt to reconsider the 'wrong' ideas is unwelcome.

This is similar to the notorious principle of democratic centralism which was popular in totalitarian communist society: a question may be discussed, but after the collective wisdom (the majority of votes) has found the 'true' decision, any discussion of alternative views is prohibited.

There are no 'true' and 'wrong' ideas or theories in reality: there are fruitful ideas and infertile ones. The history of science shows that many ideas at first declared 'wrong' have subsequently been shown to be 'true'. The main lesson of history is that nobody can ultimately decide what is the truth and what is not. The best way to proceed is not to try to 'prove' or 'disprove' a theory, but to concentrate attention on the positive aspects of any theory. Theories should be considered not as rivals but rather as complementary.

Vladimir Koliadin

Kv. 128, 162-G Tractorostroiteley Prosp.,

Kharkov 129, 310129, Ukraine 